\title{
PDGFRA promoter polymorphisms are associated with the risk of papillary thyroid cancer
}

\author{
MI-JA KIM ${ }^{1}$, SU KANG KIM ${ }^{1}$, HAE JEONG PARK ${ }^{1}$, DAE HAN CHUNG ${ }^{2}$, HYUN-KYUNG PARK ${ }^{3}$, \\ JONG SEOK LEE ${ }^{3}$, KEE HWAN KWON ${ }^{1,2}$ and JOO-HO CHUNG ${ }^{1}$ \\ ${ }^{1}$ Kohwang Medical Research Institute and Department of Pharmacology; \\ Departments of ${ }^{2}$ Otolaryngology - Head and Neck Surgery, and ${ }^{3}$ Emergency Medicine, \\ School of Medicine, Kyung Hee University, Seoul 130-701, Republic of Korea
}

Received October 17, 2011; Accepted January 13, 2012

DOI: $10.3892 / \mathrm{mmr} .2012 .784$

\begin{abstract}
Platelet-derived growth factor (PDGF) acts as a regulator in cancer development and progression. We investigated whether single nucleotide polymorphisms (SNPs) of platelet-derived growth factor receptor $\alpha$ polypeptide (PDGFRA) and platelet-derived growth factor receptor $\beta$ polypeptide (PDGFRB) genes are associated with papillary thyroid cancer (PTC) in a Korean population. Two promoter SNPs (rs6554162, -1309A/G and rs1800812, -635G/T) of PDGFRA and one promoter SNP (rs3828610, -202A/C) of PDGFRB were genotyped using direct sequencing in 93 PTCs and 212 controls. Genetic data were analyzed using the SNPAnalyzer Pro, SNPStats and Haploview programs. Two promoter SNPs (rs6554162 and rs1800812) in PDGFRA revealed significant differences between PTC and controls (for rs6554162, $\mathrm{p}=0.0018$ in the codominant model and $\mathrm{p}=0.0005$ in the dominant model; for rs1800812, $\mathrm{p}=0.016$ in the codominant model and $\mathrm{p}=0.007$ in the dominant model). In the analysis of allele frequency, we also found that the A allele of rs6554162 $(\mathrm{p}=0.004)$ and the $\mathrm{T}$ allele of rs1800812 ( $\mathrm{p}=0.029)$ were associated with PTC. Additionally, by haplotype analysis, the GG and AT haplotypes consisting of rs6554162 and rs1800812 were associated with PTC (GG, $\mathrm{p}=0.0033$; AT, $\mathrm{p}=0.0270$ ). However, rs3828610 in PDGFRB showed no significant difference between PTC and controls. The results suggest that PDGFRA promoter SNPs (rs6554162 and rs1800812) may be associated with the risk of PTC.
\end{abstract}

Correspondence to: Dr Joo-Ho Chung or Dr Kee Hwan Kwon, Kohwang Medical Research Institute, School of Medicine, Kyung Hee University, 1 Hoegi-dong, Dongdaemun-gu, Seoul 130-701, Republic of Korea

E-mail: jhchung@khu.ac.kr

E-mail: entkhkwon@empal.com

Key words: papillary thyroid cancer, platelet-derived growth factor receptor $\alpha$ polypeptide, platelet-derived growth factor receptor $\beta$ polypeptide, polymorphism

\section{Introduction}

Thyroid cancers, well known as the most common endocrine tumors in humans, have shown an increased prevalence during the past three decades $(1,2)$. They are classified pathologically as papillary, follicular, anaplastic and medullary carcinomas (2). Papillary thyroid cancer (PTC) is the most common thyroid cancer, accounting for more than $70 \%$ of cases (3). PTC is a high cure cancer with a 10 -year survival rate of $80-90 \%$ compared with other types of thyroid cancers (2). In previous studies, PTC appears to have higher heritability compared to other cancers $(4,5)$.

Platelet-derived growth factor (PDGF)/PDGF receptor (PDGFR) system contains two receptors [platelet-derived growth factor receptor $\alpha$ polypeptide (PDGFRA) and plateletderived growth factor receptor $\beta$ polypeptide (PDGFRB)] and four ligands (PDGFA, B, C and D) $(6,7)$. Expression of PDGFRs and PDGFs have crucial functions during embryogenesis, in particular in the development of connective tissue of the kidneys, blood vessels, lungs and central nervous system (7). Previous research reveals that PDGF contributes to cancer development and its progression through both autocrine and paracrine signaling mechanisms (8). Chen et al (9) showed that mRNA and protein expression of PDGFA and PDGFRA was increased in thyroid carcinoma cell lines compared to benign tissues of thyroid nodular hyperplasia. These findings indicate that the expression of PDGFRs plays a crucial role in the carcinogenesis of thyroid cells.

Genetic influence is known as the established risk factor for PTC and several studies have investigated the genetic association between PTC and candidate genes (10-12). Ozgen et al (10) reported that the $-174 \mathrm{G} / \mathrm{C}$ single nucleotide polymorphism (SNP) of interleukin-6 plays a role in the risk of thyroid cancer in Turkish patients. Lemos et al (11) demonstrated that the polymorphism of cytochrome P450 2D6 (CYP2D6) was associated with PTC. The G allele of rs3805435 or the $\mathrm{T}$ allele of rs3828599 in glutathione peroxidase 3 may exert a protective effect for differentiated thyroid cancer in an elderly population, whereas the $\mathrm{C}$ allele of rs8177412 confers an increased risk effect (12). However, not much is known concerning the variants that affect the risk of PTC. In the present study, we investigated the association between the 
promoter SNPs of PDGFRA and PDGFRB and PTC in a Korean population.

\section{Materials and methods}

Subjects. PTC patients (27 males and 66 females) and healthy individuals (107 males and 105 females) were enrolled in this study (Table I). PTC samples were obtained at the Kyung Hee University Medical Center and East-West Neomedical Center, Kyung Hee University, Seoul, Republic of Korea. Detailed clinical features, such as cancer size, number of cancers, location of cancer, extrathyroidal invasion and cervical lymph node metastasis (Table I) were available for the study patients. Control subjects were recruited through a general health check-up program and they had no clinical evidence of PTC or other severe diseases. Informed consent was obtained from all individuals according to the Declaration of Helsinki guidelines. The study was approved by the Ethics Review Committee of the Medical Research Institute, Kyung Hee University Medical Center. Age of the control group was matched to that of the PTC group.

SNP genotyping of PDGFRA and PDGFRB promoters. We searched SNPs in the promoter region of PDGFRA and PDGFRB using the SNP database (www.ncbi.nlm.nih.gov/ SNP, dbSNP BUILD 135). Among SNPs in approximately the $-1.5 \mathrm{~kb}$ promoter region of the PDGFRA gene, SNPs with $<0.05$ minor allele frequency (MAF) (rs78893477, rs1135534, rs114307010 and rs114960897) and SNPs without genotype frequency data (rs1800809, rs1800810 and rs1800813) were excluded. Similarly, for PDGFRB promoter SNPs, SNPs with <0.05 MAF (rs4705416, rs114597552, rs7719480, rs56245821, rs114597323 and rs76690893) and SNP without genotype frequency data (rs73277779) were also excluded. Finally, we selected two SNPs (rs6554162, -1309A/G and rs1800812, $-635 \mathrm{G} / \mathrm{T})$ from the PDGFRA gene and one SNP (rs3828610, -202A/C) from the PDGFRB gene. MAF of rs6554162, rs1800812 and rs3828610 was 0.3943, 0.2615 and 0.4698 , respectively (dbSNP BUILD 135). Genomic DNA was extracted from blood samples collected in EDTA using a Roche DNA Extraction kit (Roche, Indianapolis, IN, USA). Genomic DNA was amplified using the following primers for rs6554162 (sense, 5'-GCCTAAAAACCCGGTTCTCAAC-3'; antisense, 5'-ACAGGGGAGAGTCACAGAGAAG-3'; 382 bp), rs1800812 (sense, 5'-TCCAAGGAATTCGTAGGGTTC-3'; antisense, 5'-TTGTTTTGTGTGGTGTGTGTGT-3'; 317 bp) and rs3828610 (sense, 5'-GAGGCACTGAGAAATGATCTCC-3'; antisense, 5'-CTCAGGAGCTCACACCACTATG-3'; 405 bp). PCR products were sequenced using an ABI PRISM 3730XL analyzer (PE Applied Biosystems, Foster City, CA, USA) and sequence data were analyzed by SeqManII software (DNASTAR Inc., Madison, WI, USA).

Statistical analysis. To analyze the genetic data, SNPAnalyzer (Istech Inc., Goyang, Republic of Korea) and SNPStats (http:// bioinfo.iconcologia.net/index.php? module=Snpstats) were used. A linkage disequilibrium (LD) block was tested using Haploview version 4.2 (Broad Institute, Cambridge, MA, USA) (13). Multiple logistic regression models (codominant, dominant and recessive) were performed for odds ratio (OR),
Table I. Clinical characteristics of the study population.

\begin{tabular}{|c|c|c|}
\hline & PTC & Control \\
\hline Age $($ mean $\pm \mathrm{SD})$ & $53.0 \pm 11.8$ & $55.4 \pm 5.9$ \\
\hline Male/female & $27 / 66$ & $107 / 105$ \\
\hline $\begin{array}{c}\text { Cancer size } \\
<1 / \geq 1 \mathrm{~cm}\end{array}$ & $50 / 43$ & \\
\hline $\begin{array}{l}\text { No. of cancers } \\
\text { Unifocality/multifocality }\end{array}$ & $63 / 30$ & \\
\hline $\begin{array}{l}\text { Location of cancer } \\
\text { One lobe/both lobes }\end{array}$ & $67 / 26$ & \\
\hline $\begin{array}{l}\text { Extrathyroidal invasion } \\
+/-\end{array}$ & $46 / 47$ & \\
\hline $\begin{array}{l}\text { Cervical lymph node metastasis } \\
+/-\end{array}$ & $25 / 68$ & \\
\hline
\end{tabular}

PTC, papillary thyroid cancer.

$95 \%$ confidence interval (CI) and p-value (14). Analysis of data was performed using SPSS 18.0 statistical software (SPSS Inc., Chicago, IL, USA). Statistical significance was set at $\mathrm{p}<0.05$.

\section{Results}

Sample power. To test PDGFRA and PDGFRB as potential candidate genes in the development of PTC, we sequenced two promoter SNPs (rs6554162 and rs1800812) in PDGFRA and one promoter SNP (rs3828610) in PDGFRB. These three SNPs were polymorphic and the genotype distributions of the SNPs were in Hardy-Weinberg equilibrium ( $p>0.05$, data not shown). The power of the sample size was calculated to verify the data using a genetic power calculator (http://pngu.mgh.harvard. $\mathrm{edu} / \sim$ purcell/gpc/cc2.html). In this study, sample power of each SNP was 0.789 for rs6554162 $(\alpha=0.05$; genotype relative risk 2-fold; number of cases for $70 \%$ power, 75$), 0.773$ for rs 1800812 ( $\alpha=0.05$; genotype relative risk 2 -fold; number of cases for $70 \%$ power, 78) and 0.815 for $\mathrm{rs} 3828610(\alpha=0.05$; genotype relative risk 2-fold, number of cases for $70 \%$ power 70 ). Therefore, our results had relatively statistical confidence.

Genetic association between the promoter SNPs of PDGFRA and PDGFRB and PTC. As shown in Table II, rs6554162 of PDFGRA revealed significant differences between the PTC group and the control group in the codominant $(\mathrm{p}=0.0018$, $\mathrm{OR}=0.49,95 \% \mathrm{CI} 0.31-0.78)$ and the dominant models $(\mathrm{p}=0.0005, \mathrm{OR}=0.39,95 \% \mathrm{CI} 0.23-0.68)$. In the analysis of allele frequency, the A allele of rs6554162 was associated with PTC $(p=0.004, O R=0.52,95 \%$ CI 0.33-0.81), and the frequency of the A allele was decreased in PTC patients, compared to control subjects $(16.1 \%$ in the PTC group and $27.1 \%$ in the control group). The rs1800812 of PDFGRA was also significantly associated with $\mathrm{PTC}$ in the codominant $(\mathrm{p}=0.016, \mathrm{OR}=0.51,95 \% \mathrm{CI} 0.29-0.90)$ and the dominant models $(\mathrm{p}=0.007, \mathrm{OR}=0.44,95 \%$ CI $0.23-0.82)$. We also found an association between the T allele of rs1800812 and PTC $(p=0.029, \mathrm{OR}=0.54,95 \%$ CI $0.31-0.94)$. The frequency of the 
Table II. Genotype and allele frequencies of PDGFRA and PDGFRB promoter polymorphisms in PTC patients and control subjects.

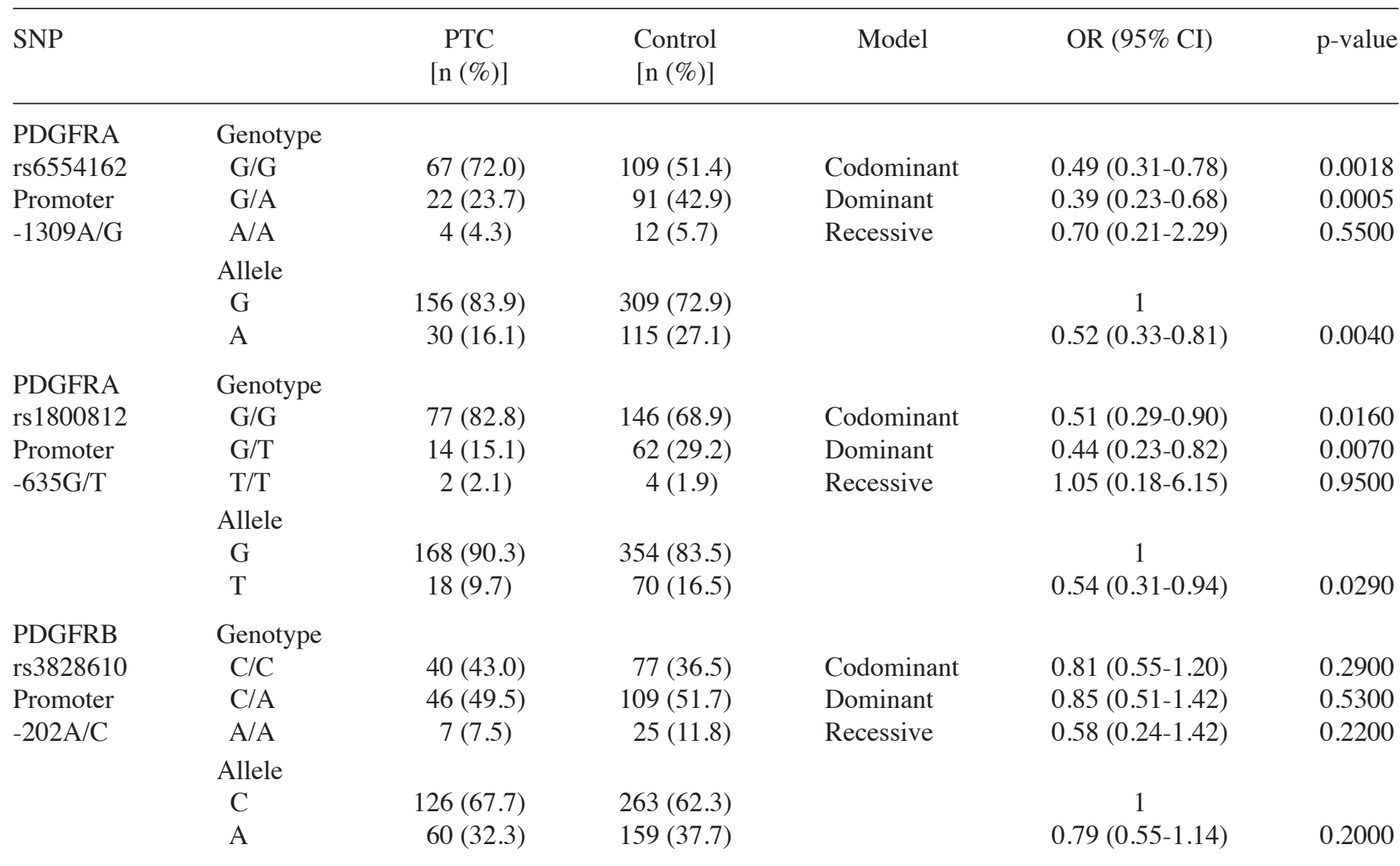

PDGFRA, platelet-derived growth factor receptor $\alpha$ polypeptide; PDGFRB, platelet-derived growth factor receptor $\beta$ polypeptide; SNP, single nucleotide polymorphism; PTC, papillary thyroid cancer; OR, odds ratio; 95\% CI, 95\% confidence interval; p-values were from logistic regression analysis with the codominant, dominant and recessive models controlling age and gender as covariates.

Table III. Haplotype analysis of PDGFRA promoter polymorphisms in PTC patients and control subjects.

\begin{tabular}{|c|c|c|c|c|c|c|c|c|}
\hline \multirow[t]{2}{*}{ Haplotype } & \multirow[t]{2}{*}{ PTC frequency } & \multirow[t]{2}{*}{ Control frequency } & \multicolumn{2}{|c|}{ PTC } & \multicolumn{2}{|c|}{ Control } & \multirow[t]{2}{*}{ Chi-square test } & \multirow[t]{2}{*}{$\mathrm{p}$-value } \\
\hline & & & + & - & + & - & & \\
\hline GG & 0.8387 & 0.7288 & 156 & 30 & 309 & 115 & 8.623 & 0.0033 \\
\hline AT & 0.0968 & 0.1651 & 18 & 168 & 70 & 354 & 4.888 & 0.0270 \\
\hline $\mathrm{AG}$ & 0.0645 & 0.1061 & 12 & 174 & 45 & 379 & 2.643 & 0.1040 \\
\hline
\end{tabular}

PDGFRA, platelet-derived growth factor receptor $\alpha$ polypeptide; PTC, papillary thyroid cancer. Haplotypes comprise rs6554162 and rs1800812.

T allele in the PTC group $(9.7 \%)$ was lower than that of the control group (16.5\%). However, rs3828610 of PDGFRB was not associated with PTC (Table II).

We also performed a further analysis according to the clinical symptoms of PTC (cancer size, number of cancers, location of cancer, extrathyroidal invasion and cervical lymph node metastasis). However, we did not find any association with the tested SNPs of PDGFRA and PDGFEB (data not shown). As shown in Fig. 1, the LD block was constructed between rs6554162 and rs1800812 of PDGFRA ( $\left.D^{\prime} \geq 0.95, r^{2} \geq 0.8\right)$ by the Gabriel method (15). In the analysis of haplotypes, GG and

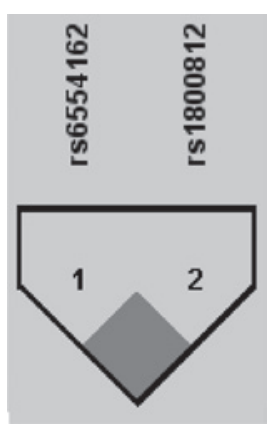

Figure 1. Linkage disequilibrium block consisting of rs6554162 and rs1800812. 
AT were associated with PTC (GG, Chi-square test 8.623, $\mathrm{p}=0.0033$; AT, Chi-square test $4.888, \mathrm{p}=0.0270$ ) (Table III).

\section{Discussion}

In this study, two promoter SNPs (rs6554162 and rs1800812) of PDGFRA were associated with PTC in a Korean population. In particular, we found that the frequencies of the A allele of rs6554162 and the $\mathrm{T}$ allele of rs1800812 were decreased in the PTC group, compared to the control group. Moreover, the GG and AT haplotypes consisting of rs6554162 and rs1800812 were also associated with PTC, respectively. However, rs3828610 of PDGFRB was not associated with PTC.

Several researchers investigated the genetic association between polymorphisms of PDGFRA or PDGFRB and several diseases (16-18). Wu et al (16) reported that rs1800810 SNP of PDGFRA was associated with the severity and allergic status of childhood asthma. De Bustos et al (17) revealed that a PDGFRA promoter polymorphism, which disrupts the binding of ZNF148, was associated with primitive neuroectodermal tumors and ependymomas. Kim et al (18) reported that the promoter SNPs (rs3756314, rs3756312 and rs3756311) of PDGFRB were associated with schizophrenia. Although we excluded above the promoter SNPs of PDGFRA and PDGFRB according to the data of dbSNP BUILD 135, two promoter SNPs (rs6554162, -1309A/G and rs1800812, -635G/T) selected in the present study were also associated with PTC. The results raise the possibility that PDGFRA promoter SNPs may contribute to the development of PTC.

To find whether the promoter SNPs affect transcription factors, the online program AliBaba 2.1 (http://www.generegulation.com/pub/programs/alibaba2) was used. At the rs6554162 SNP site in PDGFRA, the G-containing sequences bind with USF transcription factor, but USF disappears in the A-containing sequences. At the rs1800812 SNP site in PDGFRA, the G-containing sequences interact with NF1 and YY1, but these transcription factors disappear in the T-containing sequences. Assuming the change of transcript factors according to variants of SNPs, these promoter SNPs may influence gene and protein expression of PDGFRA. In addition, we compared genotype frequencies of rs6554162, rs1800812 and rs3828610 in other populations. The human SNP database (dbSNP BUILD 135) presents frequencies of the genotype for rs6554162 (A/A:A/G:G/G; European, 0.106:0.389:0.504; Sub-Saharan African, 0.381:0.504:0.115; Japanese, 0.093:0.442:0.465; Chinese, 0.116:0.419:0.465; Korean in this study, 0.057:0.429:0.514), rs1800812 (G/G:G/ T:T/T; European, 0.583:0.367:0.050; Sub-Saharan African, 0.500:0.414:0.086; Japanese, 0.591:0.364:0.045; Chinese, 0.568:0.364:0.068; Korean in this study, 0.689:0.292:0.019), and rs3828610 (C/C:A/T:A/A; European, 0.434:0.472:0.094; Sub-Saharan African, 0.021:0.362:0.617; Japanese, 0.211:0.447:0.342; Chinese, 0.125:0.700:0.175; Korean in this study, 0.365:0.517:0.118). Allele frequencies of rs6554162 in a Korean population $(\mathrm{G}, 0.729 ; \mathrm{A}, 0.271)$ are similar in pattern to those in Chinese (G, 0.674; A, 0.326) and Japanese (G, 0.686; A, 0.314) and allele frequencies of rs1800812 in Korean population $(\mathrm{G}, 0.835 ; \mathrm{T}, 0.165)$ are similar in pattern to those in Chinese ( $\mathrm{G}, 0.750$; $\mathrm{T}, 0.250)$ and Japanese $(\mathrm{G}, 0.773$; $\mathrm{T}, 0.227$ ). Also, allele frequencies of rs 3828610 in a Korean population $(\mathrm{A}, 0.377 ; \mathrm{C}, 0.623)$ are similar in pattern to those in Chinese (A, 0.475; C, 0.525) and Japanese (G, 0.434; T, $0.566)$ popoulations, respectively.

In conclusion, we found that two promoter SNPs (rs6554162 and rs1800812) of PDGFRA were associated with PTC. Our results indicate that PDGFRA may contribute to the susceptibility of PTC in a Korean population. Further studies in different populations or other SNPs of PDGFRA are required.

\section{Acknowledgements}

This study was supported by a grant from the Kyung Hee University in 2010 (KHU-20100112). The authors are grateful to Eri Kwon for the language editing.

\section{References}

1. Sherman SI: Thyroid carcinoma. Lancet 361: 501-511, 2003.

2. Caron NR and Clark OH: Papillary thyroid cancer. Curr Treat Options Oncol 7: 309-319, 2006.

3. Sakorafas GH, Giotakis J and Stafyla V: Papillary thyroid microcarcinoma: a surgical perspective. Cancer Treat Rev 31: 423-438, 2005.

4. Fagin JA: Familial nonmedullary thyroid carcinoma - the case for genetic susceptibility. J Clin Endocrinol Metab 82: 342-344, 1997.

5. Goldgar DE, Easton DF and Cannon-Albright LA: Systematic population-based assessment of cancer risk in first-degree relatives of cancer probands. J Natl Cancer Inst 86: 1600-1608, 1994.

6. Dai Y: Platelet-derived growth factor receptor tyrosine kinase inhibitors: a review of the recent patent literature. Expert Opin Ther Pat 20: 885-897, 2010.

7. Appelmann I, Liersch R, Kessler T, Mesters RM and Berdel WE: Angiogenesis inhibition in cancer therapy: platelet-derived growth factor (PDGF) and vascular endothelial growth factor (VEGF) and their receptors: biological functions and role in malignancy. Recent Results Cancer Res 180: 51-81, 2010.

8. Andrae J, Gallini R and Betsholtz C: Role of platelet-derived growth factors in physiology and medicine. Genes Dev 22: 1276-1312, 2008

9. Chen KT, Lin JD, Liou MJ, Weng HF, Chang CA and Chan EC: An aberrant autocrine activation of the platelet-derived growth factor alpha-receptor in follicular and papillary thyroid carcinoma cell lines. Cancer Lett 231: 192-205, 2006.

10. Ozgen AG, Karadeniz M, Erdogan M, Berdeli A, Saygili F and Yilmaz C: The (-174) G/C polymorphism in the interleukin-6 gene is associated with risk of papillary thyroid carcinoma in Turkish patients. J Endocrinol Invest 32: 491-494, 2009.

11. Lemos MC, Carrilho F, Rodrigues F, et al: Genetic polymorphism of CYP2D6 influences susceptibility to papillary thyroid cancer. Clin Endocrinol (Oxf) 67: 180-183, 2007.

12. Lin JC, Kuo WR, Chiang FY, et al: Glutathione peroxidase 3 gene polymorphisms and risk of differentiated thyroid cancer. Surgery 145: 508-513, 2009.

13. Barrett JC, Fry B, Maller J and Daly MJ: Haploview: analysis and visualization of LD and haplotype maps. Bioinformatics 21 : 263-265, 2005.

14. Jung KH, Ban JY, Kim HJ, et al: Genetic association study of THRB polymorphisms with obesity in Korean population. Mol Cell Toxicol 4: 124-131, 2008.

15. Gabriel SB, Schaffner SF, Nguyen H, et al: The structure of haplotype blocks in the human genome. Science 296: 2225-2229, 2002.

16. Wu LS, Tan CY, Wang LM, Lin CG and Wang JY: Variant in promoter region of platelet-derived growth factor receptor-alpha (PDGFR alpha) gene is associated with the severity and allergic status of childhood asthma. Int Arch Allergy Immunol 141: 37-46, 2006.

17. De Bustos C, Smits A, Strömberg B, Collins VP, Nistér M and Afink G: A PDGFRA promoter polymorphism, which disrupts the binding of ZNF148, is associated with primitive neuroectodermal tumours and ependymomas. J Med Genet 42: 31-37, 2005

18. Kim HJ, Kim MH, Choe BK, et al: Genetic association between 5'-upstream single-nucleotide polymorphisms of PDGFRB and schizophrenia in a Korean population. Schizophr Res 103: 201-208, 2008. 